\title{
Delocalization by Disorder in Layered Systems
}

\author{
Dmitrii L. Maslov ${ }^{a}$, Vladimir I. Yudson ${ }^{b}$, Andres M. Somoza ${ }^{c}$, and Miguel Ortuño ${ }^{c}$ \\ ${ }^{a}$ Department of Physics, University of Florida, P. O. Box 118440, Gainesville, FL 32611-8440 \\ ${ }^{b}$ Institute for Spectroscopy, Russian Academy of Sciences, Troitsk, Moscow region, 142190, Russia \\ ${ }^{c}$ Departamento de Física-CIOyN, Universidad de Murcia, Murcia 30.071, Spain
}

(Dated: October 30, 2018)

\begin{abstract}
Motivated by anomalously large conductivity anisotropy in layered materials, we propose a simple model of randomly spaced potential barriers (mimicking stacking faults) with isotropic impurities in between the barriers. We solve this model both numerically and analytically, by utilizing an exact solution for the conductivity of a one-dimensional (1D) disordered system. In the absence of bulk disorder, electron motion in the out-of-plane direction is localized. Bulk disorder destroys 1D localization. As a result, the out-of-plane conductivity is finite and scales linearly with the scattering rate by bulk impurities until planar and bulk disorder become comparable. The ac out-of-plane conductivity is of a manifestly non-Drude form, with a maximum at the frequency corresponding to the scattering rate by potential barriers.
\end{abstract}

PACS numbers: 72.15.Rn,73.20.Jc,73.21.-b

It is usually the case that cleaner metals are better conductors. In the semiclassical, phase-incoherent regime of transport, this happens simply because stronger disorder means a shorter scattering time; in the phase-coherent regime, stronger disorder enhances Anderson localization thereby reducing the conductivity even further. It is also commonly believed that localization can be destroyed only by inelastic scattering. In this Letter, we propose and analyze a simple model with two types of disorder which defies these notions. We show that an increase in one type of disorder leads to a destruction of the Anderson-localized state and, consequently, to a increase in the conductivity in one direction.

The model consists of planar barriers located at random spacings to each other and isotropic impurities distributed randomly in between the barriers (see Fig. 1). This model is motivated by some well-known but hitherto
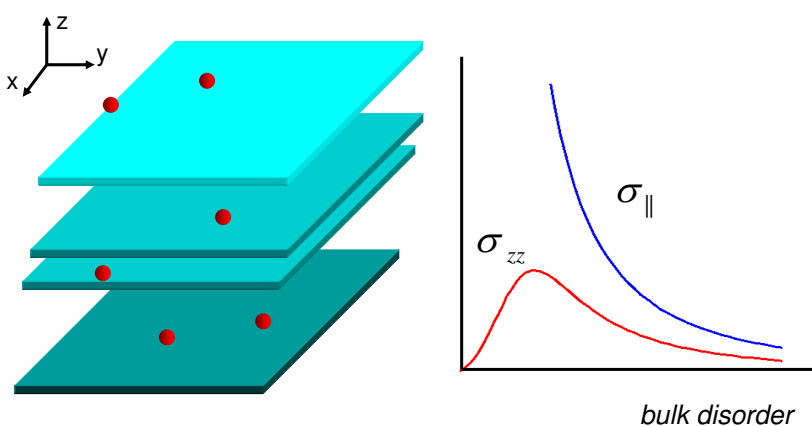

FIG. 1: (Color online) Left: a system of randomly spaced parallel potential barriers and randomly distributed isotropic impurities. Right: expected dependences of the in- and outof-plane conductivities on bulk disorder.

unexplained peculiarities of electron transport in layered conductors. In the band picture, the conductivity in a certain crystallographic direction scales with the inverse effective mass in this direction. In many cases, however, the observed ratio of the in-plane and out-of-plane conductivities exceeds the (inverse) ratio of the effective masses by several orders of magnitude. A well-known case of such an anomaly is graphite, where the conductivity ratio exceeds the mass ratio by 2-3 orders of magnitude [1], but other materials, e.g., $\mathrm{NaCo}_{2} \mathrm{O}_{4}$ [2], cuprates [3], etc., also provide examples of this behavior. Stacking faults, e.g., "wrong" planes violating Bernal stacking of graphene sheets in graphite, have been proposed to be responsible for abnormally large conductivity anisotropy long time ago [4]; however, little attention has been paid to localization of electrons by an array of faults.

We consider a system of electrons with separable but otherwise arbitrary spectrum $\varepsilon\left(\vec{k}_{\|}, k_{z}\right)=\varepsilon_{\| \mid}\left(\vec{k}_{\| \mid}\right)+$ $\varepsilon_{z}\left(k_{z}\right)$, subject to two types of random potential: the $1 \mathrm{D}$ potential of the barriers, $U(z)$, and the $3 \mathrm{D}$ potential of isotropic impurities, $V(\vec{r})$. In the absence of bulk disorder, the in- and out-of-planes degrees of freedom separate. Accordingly, the electron wave function is factorized as $\Psi\left(\vec{r}_{\|}, z\right)=\varphi\left(\vec{r}_{\|}\right) \chi(z)$, with $\chi(z)$ satisfying an effectively $1 \mathrm{D}$ Schroedinger equation $\left[\varepsilon_{z}\left(-i \partial_{z}\right)+U(z)\right] \chi(z)=\left(E-\varepsilon_{\|}\left(\vec{k}_{\|}\right)\right) \chi(z)$, where $\vec{k}_{||}$is the (quasi) momentum along the planes. All states of such a system are localized in the $z$-direction by infinitesimally weak disorder. Therefore, the $d c$ conductivity across the planes, $\sigma_{z z}$, is zero. On the other hand, since barriers do not affect the electron motion along the planes, the in-plane conductivity, $\sigma_{\|}$, is infinite. Bulk disorder mixes the in- and out-of-planes degrees of freedom, so that the separation of variables is no longer possible. Therefore, 1D localization in the $z$ direction is destroyed, and $\sigma_{z z}$ increases with bulk disorder, as long as it remains weaker than the planar one. When two disorders become comparable, $\sigma_{z z}$ reaches a maximum 
and decreases upon a further increase in bulk disorder in accord with the Drude formula [5]. At the same time, $\sigma_{\|}$decreases monotonously with bulk disorder. A sketch of expected dependences of $\sigma_{z z}$ and $\sigma_{\|}$on 3D disorder is presented in Fig. 1 (right). In the rest of the paper, we confirm this simple picture both numerically, by calculating $\sigma_{z z}$ in the Anderson model, and analytically, by exploiting the Berezinskii solution of the 1D localization problem.

Numerically, we study the Anderson model with nearest-neighbor hopping (set to unity to fix the energy scale) on a cubic lattice (of unit spacing)

$$
H=-\sum_{\mathbf{i}, \mathbf{j}} a_{\mathbf{j}}^{\dagger} a_{\mathbf{i}}+\sum_{\mathbf{i}} \epsilon_{\mathbf{i}} a_{\mathbf{i}}^{\dagger} a_{\mathbf{i}}+\text { H.c. }
$$

Here, the on-site energy $\epsilon_{\mathbf{i}}=\phi_{\mathbf{i}}+\eta_{i_{z}}$ and $\mathbf{i}=\left(i_{x}, i_{y}, i_{z}\right)$. The first term, $\phi_{\mathbf{i}}$, is the standard (bulk) disorder term which is chosen independently for each site in the interval $\left(-W_{\mathrm{B}} / 2, W_{\mathrm{B}} / 2\right)$ with uniform probability. The second term, $\eta_{i_{z}}$, describing planar disorder, is chosen as $-W$ with probability $p$ and as $W$ with probability $1-p$. For all results reported in this paper, $p=1 / 2$. The simulations are done at the energy equal to 0.1 , to avoid the center of the band. We employ the recursive Green's function technique [6] with periodic boundary conditions in the directions transverse to the $z$-axis. The out-ofplane conductance $G_{z z}$ is equal to $2 e^{2} T / h$, where $T$ is the transmission coefficient between two wide leads. The simulations were performed for cubic samples of sizes $L$ up to 35 lattice spacings. The bandwidths of planar disorder $W$ were chosen as $1,1.5,2,2.5$ and 3 , which corresponds to localization lengths between roughly 2 and 15 lattice spacings, in the absence of bulk disorder. The bandwidth of bulk disorder $W_{\mathrm{B}}$ ranged in between 0 and 18. We have averaged $\log G_{z z}$ for $10^{3}$ samples for each set of parameters. Crystalline anisotropy can be simply accounted for in simulations; however, the conductance is already anisotropic due to anisotropy of disorder even on a cubic lattice.

Figure 2 shows $\tilde{G}_{z z} \equiv \exp \left\{\left\langle\ln G_{z z}\right\rangle\right\}$ as a function of bulk disorder for several values of planar disorder. As expected, an increase in bulk disorder leads first to an in increase in $\tilde{G}_{z z}$ followed by a subsequent decrease. The position of the peak depends on planar disorder but is almost independent of $L$. We checked that the conductance scales linearly with $L$ for most of the range of parameters represented in Fig. 2, so that we are in the diffusive regime. Specifically, the diffusive regime begin when the conductance becomes larger than $2 e^{2} / h$ and continue up to the 3D Anderson transition (not shown in Fig. 22).

Figure 3 shows the collapse of the data for the conductivity, $\sigma_{z z}=\tilde{G}_{z z} / L$, on a double-logarithmic plot. Three sets of curves corresponds to three values of planar disorder: $W=1.5$ (upper set), $W=2$ (middle set) and $W=2.5$ (lower set). Within each set, the conductivity was computed for different values of $L$, as indicated in

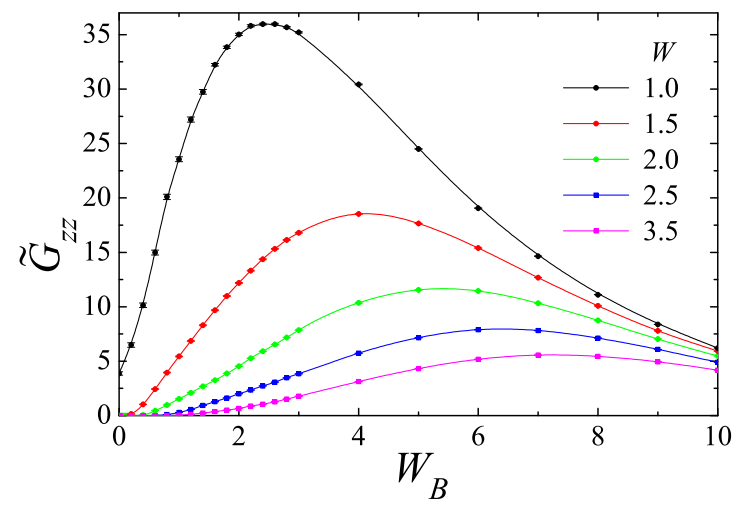

FIG. 2: (Color online) Out-of-plane conductance versus the bandwidth of bulk disorder $W_{\mathrm{B}}$ for a range of values of planar disorder $W$, as shown in the figure, and $L=30$.

the legend. The straight line has a slope equal to two. This scaling is confirmed by the analytic solution of the model, described below.

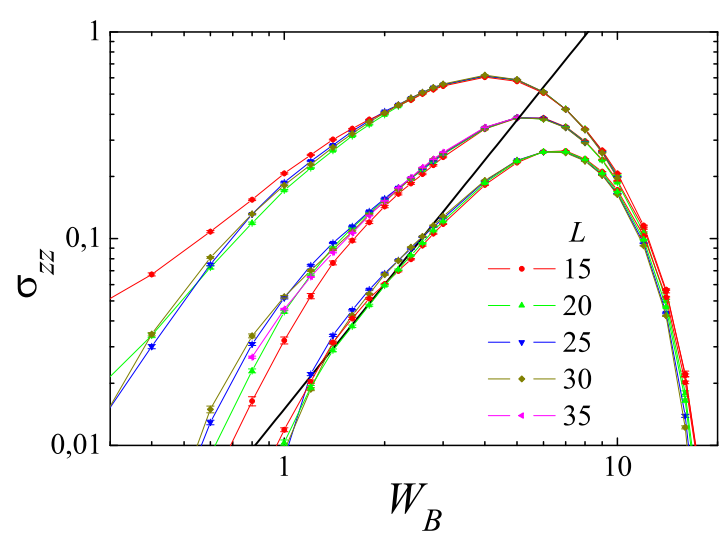

FIG. 3: (Color online) Out-of-plane conductivity versus the bandwidth of bulk disorder $W_{\mathrm{B}}$ on a double logarithmic scale for a range of system sizes, as shown in the figure, and three values of planar disorder: $W=1.5$ (upper set), $W=2$ (middle set), and $W=2.5$ (lower set).

To solve the problem analytically, we adopt the deltacorrelated forms for both types of disorder $\langle U(z) U(0)\rangle=$ $\gamma_{z} \delta(z)$ and $\langle V(\vec{r}) V(0)\rangle=\gamma \delta(\vec{r})$, and assume that bulk disorder is weaker than planar one, i.e., $1 / \tau \equiv$ $2 \pi \nu_{3}\left(E_{F}\right) \gamma \ll 1 / \tau_{z}=2 \pi \nu_{1}\left(E, \vec{k}_{||}\right) \gamma_{z}$, while planar disorder is weak in a sense that $E_{F} \tau_{z} \gg 1$. Here, $\nu_{3}$ is the $3 \mathrm{D}$ density of states and $\nu_{1}$ is the $1 \mathrm{D}$ density of states at fixed value of $\vec{k}_{||}$per one spin orientation. In the absence of bulk disorder, our problem reduces to the 1D case 
with the velocity $v_{z}=\left|\partial \varepsilon\left(\vec{k}_{\|}, k_{z}\right) / \partial k_{z}\right|_{k_{z}=k_{z F}\left(\vec{k}_{||}\right)}$, where $k_{z F}\left(\vec{k}_{\|}\right)$is a positive root of the equation $\varepsilon\left(\vec{k}_{\|}, k_{z}\right)=E_{F}$, and the scattering time $\tau_{z}$ being functions of $\vec{k}_{\|}$. The result for the $a c$ conductivity of a strictly 1D disordered system, surmised first by Mott [7] and derived rigorously by Berezinskii [8] reads

$$
\sigma^{1 D}(\omega)=\frac{16 e^{2} v_{z} \tau_{z}}{\pi}\left[-i \zeta(3) \omega \tau_{z}+2 \tau_{z}^{2} \omega^{2} \ln ^{2}\left(\omega \tau_{z}\right)\right],
$$

for $\omega \tau_{z} \ll 1$. (The numerical coefficient in the imaginary part was corrected in Refs. [9, 10]). The out-of-plane conductivity of a 3D sample with $V=0$ is obtained from Eq. (2) by summing over $\vec{k}_{\|}: \sigma_{z z}(\omega)=\int d^{2} k_{\|} \sigma^{1 D}(\omega) /(2 \pi)^{2}$. As expected, $\sigma_{z z}(0)=0$.

In the presence of both types of disorder, $\sigma_{z z}$ is given by the Kubo formula

$$
\begin{aligned}
& \sigma_{z z}(\omega)=\frac{e^{2}}{2 \pi} \frac{1}{\mathcal{A}^{3}} \sum_{\vec{k}_{\| \mid}, \vec{k}_{\|}^{\prime}} \int d z^{\prime} \\
& \times\left\langle\left\langle v_{z} \mathcal{G}_{+}^{R}\left(\vec{k}_{\|}, z ; \vec{k}_{\|}^{\prime}, z^{\prime}\right) v_{z}^{\prime} \mathcal{G}_{-}^{A}\left(\vec{k}_{\|}^{\prime}, z^{\prime} ; \vec{k}_{\|}, z\right)\right\rangle_{\mathrm{p}}\right\rangle_{\mathrm{b}},
\end{aligned}
$$

where $\mathcal{G}_{ \pm}^{R(A)}=\mathcal{G}^{R(A)}\left(\vec{k}_{\|}, z ; \vec{k}_{\|}^{\prime}, z^{\prime} ; E_{F} \pm \omega / 2\right)$ is an exact retarded (advanced) electron Green's function in the mixed $\vec{k}_{\|}-z$ representation for a given disorder realization, $\mathcal{A}$ is the sample area in the lateral direction, and $\langle\ldots\rangle_{\mathrm{b}, \mathrm{p}}$ denotes averaging over bulk and planar disorders, correspondingly. The diagram for $\sigma_{z z}$ is shown in Fig. [ 4 on the left. To leading order in $\gamma$, the conductivity $\sigma_{z z}^{(1)}$ averaged over bulk disorder is given by the sum of the two diagrams in the first row of Fig. 4, where thick solid lines denote Green's functions in the absence of bulk disorder, $\mathcal{G}_{\mathrm{p}}^{R(A)}\left(z, z^{\prime} ; \vec{k}_{\|} ; E\right)$, and zigzags denote the correlation function of bulk disorder. There are no vertex corrections for the case of delta-correlated bulk disorder. The first (second) diagram in the first row of Fig. 4 is obtained by replacing the exact Green's function by $\gamma \int_{z_{1}, \vec{p}_{||}} \mathcal{G}_{\mathrm{p}}^{R(A)}\left(z, z_{1} ; \vec{k}_{||}\right) \mathcal{G}_{\mathrm{p}}^{R(A)}\left(z_{1}, z_{1} ; \vec{p}_{||}\right) \mathcal{G}_{\mathrm{p}}^{R(A)}\left(z_{1}, z^{\prime} ; \vec{k}_{||}\right)$.

Subsequent averaging over planar disorder is simplified dramatically by noticing that the effective energies $E-\varepsilon_{\|}\left(\vec{k}_{\|}\right)$of the Green's functions depend on a particular value of $\vec{k}_{\|}$. For short-range bulk disorder, the momentum $\vec{p}_{\|}$of the Green's function below the zigzag line differs considerably from the momentum $\vec{k}_{\|}$ in the rest of the diagram. This means that the typical difference of corresponding energies is of order $E_{F}$, i.e., much greater than $1 / \tau_{z}$. In this situation, one can safely neglect correlations between the Green's functions with different momenta and average $\mathcal{G}_{\mathrm{p}}\left(z_{1}, z_{1} ; \vec{p}_{\|}, E\right)$ over planar disorder independently from the rest of the diagram. As a result, we arrive at the diagrams in the second row of Fig. 4, where thick dashed lines denote the Green's function averaged over planar disorder. For weak planar disorder $\left(\begin{array}{lll}E_{F} \tau_{z} & \gg 1\end{array}\right)$,

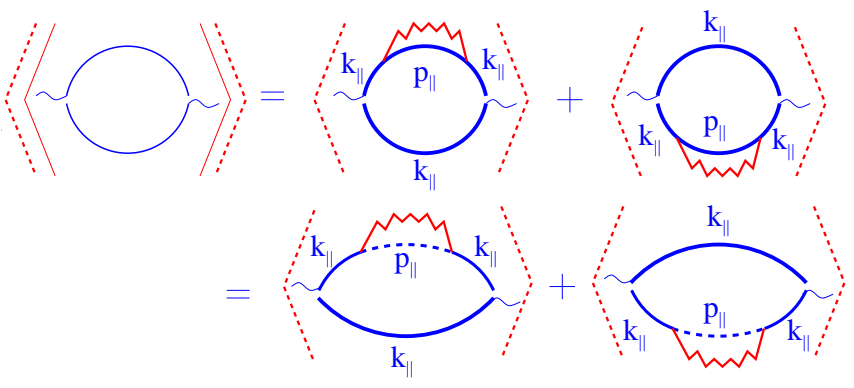

FIG. 4: (Color online) Diagrams for the out-of-plane conductivity to leading order in bulk disorder. Thin lines: exact Green's functions in the presence of both types of disorder; thick solid lines: Green's functions in the presence of planar disorder only; thick dashed lines: Green's functions averaged over planar disorder; zigzag: correlator of bulk disorder; solid and dashed brackets: averaging over planar and bulk disorder, correspondingly.

this Green's function is $\left\langle\mathcal{G}_{\mathrm{p}}^{R, A}\left(z, z ; \vec{k}_{\|} ; E\right)\right\rangle_{\mathrm{p}}=$ $\int d k_{z}\left[E-\varepsilon_{z}\left(k_{z}\right)-\varepsilon_{\|}\left(\vec{k}_{\|}\right) \pm i / 2 \tau_{z}\right]^{-1} / 2 \pi \quad$ and the corresponding self-energy insertion reduces to $\Sigma^{R(A)}\left(z, z^{\prime} ; \vec{k}_{\|} ; E\right)=\mp(i / 2 \tau) \delta\left(z-z^{\prime}\right)$. Expanding $\mathcal{G}_{\mathrm{p}}^{R, A}$ over the basis exact of eigenstates of the 1D problem, we reduce the convolution of two Green's functions, sharing the point $z_{1}$, to $\int_{z_{1}} \mathcal{G}_{\mathrm{p}}^{R, A}\left(z, z_{1} ; \vec{k}_{\|} ; E\right) \mathcal{G}_{\mathrm{p}}^{R, A}\left(z_{1}, z^{\prime} ; \vec{k}_{\|} ; E\right)=$ $-\frac{\partial}{\partial E} \mathcal{G}_{\mathrm{p}}^{R, A}\left(z, z^{\prime} ; \vec{k}_{\|} ; E\right)$. Consequently, $\sigma_{z z}^{(1)}(\omega)$ is obtained from the exact $1 \mathrm{D}$ result via

$$
\sigma_{z z}^{(1)}(\omega)=\frac{i}{\tau} \int \frac{d^{2} k_{\|}}{(2 \pi)^{2}} \frac{\partial \sigma^{1 D}(\omega)}{\partial \omega} .
$$

To obtain the $d c$ conductivity, one needs to differentiate only the imaginary part of Eq. (2). This gives

$$
\sigma_{z z}^{(1)}(0)=2 e^{2} \nu_{3}\left(E_{F}\right) D_{z z},
$$

where

$$
D_{z z}=16 \zeta(3) \frac{\left\langle v_{z}^{4}\left(\vec{k}_{\|}\right)\right\rangle_{\|}}{v_{z, \max }^{4}} \frac{l_{z, \max }^{2}}{\tau} .
$$

Here, $l_{z, \max }$ and $v_{z, \max }$ denote the maximum values of $l_{z}\left(\vec{k}_{\|}\right) \equiv v_{z} \tau_{z}$ and $v_{z}\left(\vec{k}_{\|}\right)$attained for $\vec{k}_{\|}=0$ and $\left\langle f\left(\vec{k}_{||}\right)\right\rangle_{\|}=\left(4 \pi^{2} \nu_{3}\left(E_{F}\right)\right)^{-1} \int d^{2} k_{||} \nu_{1}\left(E_{F}, \vec{k}_{||}\right) f\left(\vec{k}_{||}\right)$. The diffusion coefficient $D_{z z}$ is proportional to the ratio of the square of the localization length in the $1 \mathrm{D}$ system to the bulk scattering time. Numerically, we have found that $\sigma_{z z}(0)$ scales as the square of the bulk disorder bandwidth. This is confirmed by our analytic result since $\sigma_{z z}^{(1)}(0) \propto 1 / \tau \propto \gamma \propto B^{2}$ in the Born approximation.

Equation (6) allows for a simple physical interpretation. Bulk scattering weakly couples 1D channels of localized electrons with different $\vec{k}_{\|}$. Each scattering 
event results in a random displacement of order $l_{z}$ in the $z$-direction and results in diffusion with the coefficient $D_{z z} \sim l_{z}^{2} / \tau$. Notice that bulk disorder acts very similarly to the electron-phonon (e-ph) interaction in a strictly $1 \mathrm{D}$ system, where $\sigma^{1 D}(0) \propto 1 / \tau_{\mathrm{e}-\mathrm{ph}}[9]$. The difference between the two cases is that $\sigma^{1 D}(0)$ scales with $1 / \tau_{\mathrm{e}-\mathrm{ph}}$ only at temperatures higher than the single-level spacing within the localization length, i.e, for $T \tau_{z} \gg 1$, while at lower temperatures $\sigma^{1 D}$ is of the hopping form. The condition $T \tau_{z} \gg 1$ allows one to neglect correlations between the Green's functions in the self-energy insertions and in the rest of the diagram. In our case, these correlations can be always neglected for short-range bulk disorder, i.e., in contrast to phonon-activated transport, there is no "hopping" regime for disorder-activated transport.

Coming back to the issue of anomalously large conductivity anisotropy, it is easy to show that the inplane conductivity is given by the usual Drude formula $\sigma_{\alpha \beta}=\delta_{\alpha \beta} e^{2} \nu_{3}\left(E_{F}\right)\left\langle v_{\alpha} v_{\beta}\right\rangle_{\|} \tau$. Then the conductivity ratio can be estimated as

$$
\sigma_{\|} / \sigma_{z z} \sim\left(\left\langle v_{\|}^{2}\right\rangle_{\|} /\left\langle v_{z}^{2}\right\rangle_{\|}\right)\left(\tau / \tau_{z, \max }\right)^{2} .
$$

As an example, we consider the case of graphite with $\sigma_{\|} / \sigma_{z z}=10^{4}$ at low temperatures. A realistic band structure model of graphite [1] gives $\left\langle v_{\|}^{2}\right\rangle /\left\langle v_{z}^{2}\right\rangle \sim 140$, thus $\tau_{z} / \tau \sim 0.12$. Taking $\tau=0.5 \times 10^{-12} \mathrm{~s}$ from Ref. [1] and estimating $\left\langle v_{z}^{2}\right\rangle^{1 / 2} \sim 2 \times 10^{6} \mathrm{~cm} / \mathrm{s}$, we obtain for the mean free path due to planar disorder (stalking faults) in $l_{z} \sim 120 \AA$. This means that stalking faults are separated by about a hundred perfect planes, which is quite a realistic assumption.

Summing up higher-order diagrams with self-energy insertions due to bulk disorder amounts to replacing the exact Green's functions in Eq. (3) by $\mathcal{G}^{R(A)}\left(z, z^{\prime} ; \vec{k}_{\|} ; E \pm\right.$ $\left.\omega / 2 \pm \frac{i}{2 \tau}\right)$, which can be viewed as functions of a complex frequency. One can verify that all intermediate steps in Refs. [8] and [10] are valid for complex $\omega$ as well. Therefore, the general result for the conductivity of our model is obtained from the Berezinskii's solution as

$$
\sigma_{z z}(\omega)=\int \frac{d^{2} k_{\|}}{(2 \pi)^{2}} \sigma^{1 D}\left(\omega+\frac{i}{\tau}\right)
$$

To lowest order in $1 / \tau$, Eq. (8) reduces back to Eq. (4). Within the logarithmic accuracy of the original Berezinskii's formula, we obtain

$$
\begin{aligned}
& \operatorname{Re} \sigma_{z z}(\omega)=2 e^{2} \nu_{3}\left\langle\frac{16 l_{z}^{2}}{\tau}\left[\zeta(3)+\frac{2 \tau_{z}}{\tau}\left(\omega^{2} \tau^{2}-1\right) \ln ^{2}\left\{\omega^{2} \tau_{z}^{2}+\frac{\tau_{z}^{2}}{\tau^{2}}\right\}\right]\right\rangle_{\|} \\
& \operatorname{Im} \sigma_{z z}(\omega)=-2 e^{2} \nu_{3} \omega \tau\left\langle\frac{16 l_{z}^{2}}{\tau}\left[\zeta(3)-\frac{2 \tau_{z}}{\tau} \ln ^{2}\left\{\omega^{2} \tau_{z}^{2}+\frac{\tau_{z}^{2}}{\tau^{2}}\right\}\right]\right\rangle_{\|}
\end{aligned}
$$

These formulas are valid for an arbitrary value $\omega \tau$ but only for $\omega \tau_{z} \ll 1$ and $\tau_{z} / \tau \ll 1$. From Eq. (9a), we see that $\operatorname{Re} \sigma_{z z}(\omega)$ is almost constant for $\omega \ll \omega_{\text {cr }} \equiv$ $1 /\left(\tau_{z, \max } \tau\right)^{1 / 2}$ and increases with $\omega$ in a Mott way, as $\omega^{2} \ln ^{2} \omega$, for $\omega \gg \omega_{\text {cr }}$. At higher frequencies, $\omega \gg 1 / \tau_{z}$, $\sigma_{z z}(\omega)$ can be found perturbatively in $1 / \tau_{z}$ : the leading order result is simply a Drude formula $\sigma_{z z}(\omega) \propto$ $1 / \omega^{2} \tau_{z}-i / \omega$. Therefore, both $\operatorname{Re} \sigma_{z z}(\omega)$ and $-\operatorname{Im} \sigma_{z z}(\omega)$ have maxima at $\omega \sim 1 / \tau_{z}$. Thus, although bulk disorder destroys localization at $\omega=0$, the resulting state still has properties interpolating between those of a metal and an Anderson insulator. This prediction is amenable to a direct experimental verification.

Finally, we notice that the predictions of our model are equally well applicable to a two-dimensional (2D) case, e.g, for line barriers crossing the plane. Such a system can be realized in a $2 \mathrm{D}$ electron gas with an array of randomly spaced stripe-like gates.

In conclusion, we have shown that a system with two types of disorder-randomly spaced planar barriers and bulk impurities-exhibits quite unusual transport prop- erties. In the absence of bulk disorder, it behaves as a $1 \mathrm{D}$ insulator in the out-of-plane direction and as an ideal metal in the in-plane direction. Bulk disorder renders both conductivities finite; however, $\sigma_{z z}$ increases with bulk disorder until two disorders become comparable. For weak bulk disorder, the ratio of the conductivities may exceed the ratio of the effective masses by orders of magnitude. The $a c$ out-of-plane conductivity has a manifestly non-Drude frequency dependence with a maximum at intermediate frequencies.

We thank S. Blundell, H. Bouchiat, S. Brazovskii, K. Efetov, A. Hebard, S. Gueron, D. Gutman, N. Kirova, I. Lerner, G. Montambaux, H. Pal, P. Hirschfeld, É. Rashba, A. Schofield, S. Tongay, and I. Yurkevich for stimulating discussions. D.L.M. acknowledges the financial support from RTRA Triangle de la Physique and hospitality of the Laboratoire de Physique des Solides, Université Paris-Sud, where a part of this work was done. D.L.M. and V.I.Y. acknowledge hospitality of ICTP (Trieste). V.I.Y. acknowledges RFBR grant 09-02-01235. A.M.S and M.O. acknowledge financial support from 
the Spanish DGI, project FIS2006-11126, and Fundacion Seneca, project 08832/PI/08.

[1] N. B. Brandt, S. M. Chudinov, and Ya. G. Ponomarev, Semimetals: I. Graphite and its Compounds (NorthHolland, Amsterdam, 1988).

[2] D. J. Singh, Phys. Rev. B 61, 13397 (2000).

[3] W. E. Pickett, Rev. Mod. Phys. 61, 433 (1989).

[4] S. Ono, J. Phys. Soc. Jap. 40, 498 (1976).
[5] A further increase in bulk disorder will lead eventually to a $3 \mathrm{D}$ Anderson localization, but we are not studying this regime here.

[6] A. MacKinnon, Z. Phys. B 59, 385 (1985).

[7] N. F. Mott, Phil. Mag. 22, 7 (1970).

[8] V. L. Berezinskii, Sov. Phys. JETP 38, 620 (1974).

[9] A. A. Gogolin, V. I. Mel'nikov, and É. I. Rashba, Sov. Phys. JETP 42, 168 (1975).

[10] A. A. Abrikosov and I. A. Ryzhkin, Adv. Phys. 27, 147 (1978).

[11] Xu Du, S.-W. Tsai, D. L. Maslov, and A. F. Hebard, Phys. Rev. Lett. 94, 166601 (2005). 